\title{
Ganancia de peso diaria de machos castrados vs. machos enteros durante la recría
}

\author{
Average daily gain of intact and castrated calves during the backgrounding phase
}

\author{
Arias, R.A. ${ }^{a *}$, Pérez, Fehrmann, J.A. ${ }^{b}$, Velásquez, A.C. ${ }^{b \neq}$, Alvarado-Gilis, Ch. A. ${ }^{a}$ \\ ${ }^{a}$ Instituto de Producción Animal, Universidad Austral de Chile, Valdivia-Chile. \\ ${ }^{b}$ Escuela de Agronomía, Universidad Católica de Temuco, Chile. \\ ₹ Núcleo de Investigación en Producción Agroalimentaria, Universidad Católica de Temuco.
}

\begin{tabular}{|c|}
\hline ART ICLE INFO \\
\hline $\begin{array}{l}\text { Article history: } \\
\text { Received 12.11.14 } \\
\text { Accepted 12.03.15 }\end{array}$ \\
\hline $\begin{array}{l}\text { Keywords: } \\
\text { Beef cattle } \\
\text { Young bulls } \\
\text { Animal welfare }\end{array}$ \\
\hline $\begin{array}{l}\text { Original Research Article } \\
\text { Animal Science }\end{array}$ \\
\hline $\begin{array}{l}\text { *Corresponding author: } \\
\text { Rodrigo Arias } \\
\text { E-mail address: } \\
\text { rodrigo.arias@uach.cl }\end{array}$ \\
\hline
\end{tabular}

\section{A R T I C LE INFO}

Accepted 12.03.15

Keywords:

Beef cattle

Young bulls

Animal welfare

Original Research Article

Animal Science

Rodrigo Arias

rodrigo.arias@uach.cl

\section{A B S T R A C T}

The study was conducted in Osorno Chile, in order to assess the effect of castration on average daily gain of weaned calves during the backgrounding phase. The study considered a total of 20 weaned calves of a beef biotype whose average live weight was $189.5 \mathrm{~kg}$ ( $\pm 4.3 \mathrm{SEM}$ ). Two groups of 10 animals each were randomly assigned to two treatments consisted in calves castrated after weaned and intact calves. Both groups were handled similarly; grazing natural pastures composed mainly of Trifolium repens L., Achillea millefolium L. and Anthoxanthum odoratum L. The study was conducted as a completely randomized design, with each animal being considered as experimental and observational unit. Data were analyzed using the statistical package JMP (SAS Institute, N.C. USA) by using a covariance analysis (ANCOVA by initial body weight) with a significance level of $5 \%$. The evaluation period was 90 days and animals were weighted fortnightly. Total weight gains during the study period were 113.6 y $117.6 \mathrm{~kg}( \pm 5.7 \mathrm{SEM})$ for castrated and intact calves, respectively. No differences were observed between average daily weight gain (castrated $=1.27 \mathrm{vs}$. intact $=1.30 \mathrm{~kg} / \mathrm{d}$ $\pm 0.06 \mathrm{~kg} / \mathrm{d}$ ). We conclude that there are no productive advantages by keeping intact animals, thereby increasing the risk of stress, suffering and risk of operators to perform the castration.

\section{RESUMEN}

El objetivo del estudio fue determinar el impacto de la castración al inicio de la etapa de recría sobre su ganancia diaria de peso. Se utilizaron 20 animales de biotipo cárnico cuyo peso promedio inicial fue de 189,5 $\mathrm{kg}( \pm 4,3 \mathrm{EEM})$. La mitad fue asignada aleatoriamente a uno de los dos tratamientos, que correspondieron a teneros castrados después del destete al inicio de la recría y la otra mitad correspondió a animales enteros. Todos los animales fueron manejados de forma similar, pastoreando praderas naturales compuestas mayoritariamente por Trifolium repens L., Achillea millefolum L. y Anthoxanthum odoratum L. El diseño experimental fue completamente al azar, siendo la unidad experimental y observacional cada animal. El período de evaluación fue de 90 días y el peso de los animales fue registrado a intervalos de 15 días. Los datos fueron analizados con el paquete estadístico JMP (SAS Institute N.C., USA), utilizando un análisis de covarianza con el peso inicial como cofactor y con un nivel de significancia del 5\%. Las ganancias de peso total durante el período de estudio fueron de 113,6 y 117,6 kg ( \pm 5,7 EEM) para castrados y enteros, respectivamente. Las ganancias diarias de peso para los 90 días fueron 1,27 y 1,30 kg/d ( $\pm 0,06 \mathrm{~kg} / \mathrm{d})$ para castrados y enteros, respectivamente $(\mathrm{P}=0,79)$. Los resultados obtenidos permiten concluir que no existen ventajas productivas al mantener animales enteros en la recría, aumentando con ello el riesgo de estrés, sufrimiento y riesgo de los operarios al realizar la posterior castración.

Palabras clave: Ganado de carne, toretes, bienestar animal.

\section{INTRODUCCIÓN}

La castración de los machos en la ganadería de carne tiene por objeto reducir la agresividad y comportamiento sexual indeseado, así como también mejorar la calidad de la carne producida (Field, 1971). Por otra parte, el posicionamiento de la temática del bienestar animal y su impacto tanto en la producción como en la calidad del producto obtenido ha cobrado mayor rele- vancia durante la última década (Müller-Lindenlauf et al., 2010). Sin embargo, también ha cuestionado algunas prácticas habituales de manejo animal tales como inyecciones, descuerne y castración (Mosher et al., 2013). En este sentido, Hocquette et al. (2014) han asociado a la castración como uno de los factores extrínsecos que afectan la calidad de la carne. En tanto, Duff y Galyean (2007) definen la castración como una de las actividades que generan mayor estrés en los animales. A la fe- 
cha, una gran parte de los esfuerzos realizados en Chile referentes al bienestar animal han estado asociados al transporte del ganado, pero poco a poco se han incorporado nuevas temáticas como la castración o destete.

En Chile, una gran mayoría de los productores castran convencionalmente sus animales, antes de finalizar la etapa de recría, con al menos un mes de anticipación previo a la fecha de venta para su engorda. Lo anterior con la idea de aprovechar las hormonas naturales que el macho produce y el impacto de estas sobre la eficiencia y producción del animal en crecimiento. Sin embargo, en este punto existe controversia dado que la producción de hormonas anabólicas naturales no es significativa sino hasta después de la pubertad, la cual en promedio se alcanza a los 10 meses de vida, aún cuando existen otros factores que pueden modificar la edad de la pubertad, tales como la raza y el estado nutricional del animal (Bretschneider, 2005). En consecuencia, la castración post-puberal generaría estrés en el animal con una consecuente reducción en su respuesta productiva y un mayor tiempo de recuperación de sus heridas, lo que diluye los efectos positivos que implica dejar los animales intactos (Jago et al., 1999; Fisher et al., 2001). El dejar los animales enteros implica que la carne sea más magra y que el manejo de los mismos sea un poco más complicado, debido a los efectos de temperamento asociados a los animales enteros así como un mayor riesgo de estos a presentar altos valores de pH en la canal (Field, 1971; Knight et al., 1999a). La hipótesis a evaluar fue que la producción de testosterona de los machos enteros a esta edad no es suficiente como para generar efectos medibles en ganancia diaria de peso. Por ello, el objetivo de esta investigación fue determinar si la práctica común de castración realizada al comienzo de la etapa de recría presenta alguna desventaja en términos de ganancia diaria de peso al compararlos con machos enteros.

\section{MATERIALES Y MÉTODOS}

El ensayo fue realizado en el fundo Los Álamos, ubicado a $8 \mathrm{~km}$ al sureste de la ciudad de Osorno, de la misma comuna. Los animales utilizados correspondieron a 20 terneros destetados enteros de biotipo cárnico (híbridos Angus, Hereford y Clavel), adquiridos en la feria Ganaderos de Osorno, los que pastorearon una superficie de 20 hectáreas de praderas naturales mejoradas por un período de 90 días durante la primavera. Los animales fueron pesados a su llegada al predio $(189,5 \mathrm{~kg} \pm 4,3 \mathrm{EEM})$, posteriormente fueron asignados en forma aleatoria a uno de los dos grupos de 10 animales. Los animales castrados fueron identificados con aretes de color blanco y los animales enteros con aretes de color celeste. A todos los animales se les realizó el mismo manejo sanitario, consistente en antiparasitario vía subcutánea (Ivermectina; $1 \mathrm{~mL} / 50 \mathrm{~kg}$ de peso vivo). Los animales del grupo 1 fueron castrados el 21/09/2010 mediante método convencional (cirugía). La zona de cirugía y los utensilios fueron desinfectados mediante agua con yodo, mientras que la desinfección de la herida se efectuó con cloranfenicol spray. Los pesajes se realizaron individualmente a intervalos de 15 días durante la mañana sin destare con balanza digital (Gallagher, modelo 300), comenzando el 28/09/2010 y finalizando el 27/12/2010.

Las especies dominantes de la pradera utilizada correspondieron a trébol blanco (Trifolium repens L.), pasto oloroso (Anthoxanthum odoratum L.) y mil en ramas (Achillea millefolium L.). Al finalizar el ensayo se realizó un análisis bromatológico rutinario de la pradera en el laboratorio de INIA Remehue Osorno (22 de diciembre del 2010) observándose los resultados presentados en el Cuadro 1.

\section{Diseño experimental y análisis estadístico}

El modelo experimental utilizado correspondió a un diseño completamente al azar con dos tratamientos: animales castrados vs. enteros. El número de unidades experimentales y observacionales fue de 10 por tratamiento. Se realizaron pruebas de normalidad (ShapiroWilk) y homocedasticidad (Levene), así como un Análisis de Covarianza, utilizando el peso inicial como covariable. El nivel de significancia fue de 5\%. El modelo estadístico evaluado fue:

$$
Y_{i j}=\mu+\alpha_{i}+\mathrm{b} X_{i j}+\varepsilon_{i j}
$$

dónde: $\mu$ corresponde al promedio de la población; $\alpha$ representa el efecto del "ivo" tratamiento, b es un coeficiente de la relación entre la covariable y la variable dependiente, $X_{i j}$ es la covariable peso inicial y $\varepsilon$ es el error

Cuadro 1.Valores del análisis bromatológico de la pradera utilizada en el ensayo.

Table 1. Bromatological values of the pasture utilized in the study.

\begin{tabular}{|c|c|c|}
\hline Ítem & Valor & Valor referencial $^{1}$ \\
\hline Materia seca, \% & 16,5 & 18,52 \\
\hline Proteína cruda, \% & 19,2 & 17,88 \\
\hline $\begin{array}{l}\text { Energía metabolizable } \\
\text { (Mcal/kg MS) }\end{array}$ & 2,74 & 2,64 \\
\hline Fibra detergente neutro, $\%$ & 32,9 & 46,53 \\
\hline Cenizas, \% MS & 9,9 & 9,22 \\
\hline Valor D, \% & 75,8 & -- \\
\hline Digestibilidad in vitro, (\%) & 85,7 & -- \\
\hline
\end{tabular}

${ }^{1}$ Pradera permanente fertilizada, lechería, Osorno (Anrique et al., 2014). 
experimental asociado al $\mathrm{i}^{\text {avo }}$ animal del $\mathrm{j}^{\text {avo }}$ tratamiento. Los datos fueron analizados con el paquete estadístico JMP 11.0 (SAS Institute, N.C. USA)

\section{RESULTADOS}

El Cuadro 1 presenta el valor nutricional de la pradera. En términos generales se observa un contenido de PC que sobrepasa los requerimientos de los animales en estudio, los que según el NRC (2000) son de aproximadamente un 10 a 12\%. En éste caso se observó un valor de la pradera de 19,5\%, lo que podría explicarse por la alta densidad de plantas de trébol blanco presentes en la pradera. El valor de energía metabolizable (EM) fue 0,1 unidades superior al valor de referencia y el FDN 13,6 puntos menor. Lo anterior, sumado al alto valor de digestibildiad representa un forraje de muy buena calidad, con un alto potencial de consumo.

La Figura 1 muestra la evolución en la ganancia diaria de peso vivo de los animales, presentando ambos grupos un patrón similar. Se observó un aumento sostenido de la ganancia de peso vivo, salvo al periodo comprendido entre el 10/12/2010 y el 27/12/2010 (75 días después de la castración), en el cual se registró una baja importante en la ganancia diaria de peso de ambos grupos, pero especialmente en el grupo de animales castrados (Figura 2). Así, al parecer, la calidad de la pradera sería adecuada para lograr altas ganancias de peso. En efecto la calidad de la pradera se asemeja más bien a una pradera de la zona correspondiente al mes de octubre (Anrique et al., 2014). No obstante lo anterior, las ganancias del último periodo alcanzaron valores similares de GDP a la observada en los periodos de 30, 45 y 60 días después de la castración. Esto último producto de las mejores condiciones climáticas para el crecimiento de las praderas, pero con una diferencia de $0,36 \mathrm{~kg} /$ día en favor de los animales castrados (1,29 vs. 0,93 para castrados y enteros, respectivamente), lo que reflejaría un efecto compensatorio. Sin embargo, no se observaron diferencias significativas para las GDP de los novillos en ninguno de los períodos evaluados, incluido el período 5 cuyos promedios de GDP fueron de $-0,03 \mathrm{~kg} / \mathrm{d}$ y $0,35 \mathrm{~kg} / \mathrm{d}$ para animales castrados y enteros $( \pm 0,26 \mathrm{EEM}$; $=0,32)$.

Como era esperable las ganancias de peso vivo fueron disminuyendo en la medida que avanzaba la temporada de estudio (Figura 2). En tanto, la Figura 3 muestra la ganancia diaria de peso a los 90 días para ambos tratamientos, no encontrándose diferencias entre estos $(\mathrm{P}=0,79)$, pero en ambos tratamientos con ganancias por sobre el $1,2 \mathrm{~kg} / \mathrm{d}$.

\section{DISCUSIÓN}

La calidad nutritiva de la pradera presentó valores similares a los reportados en la literatura (Anrique et al., 2014) e incluso superiores en términos de EM y

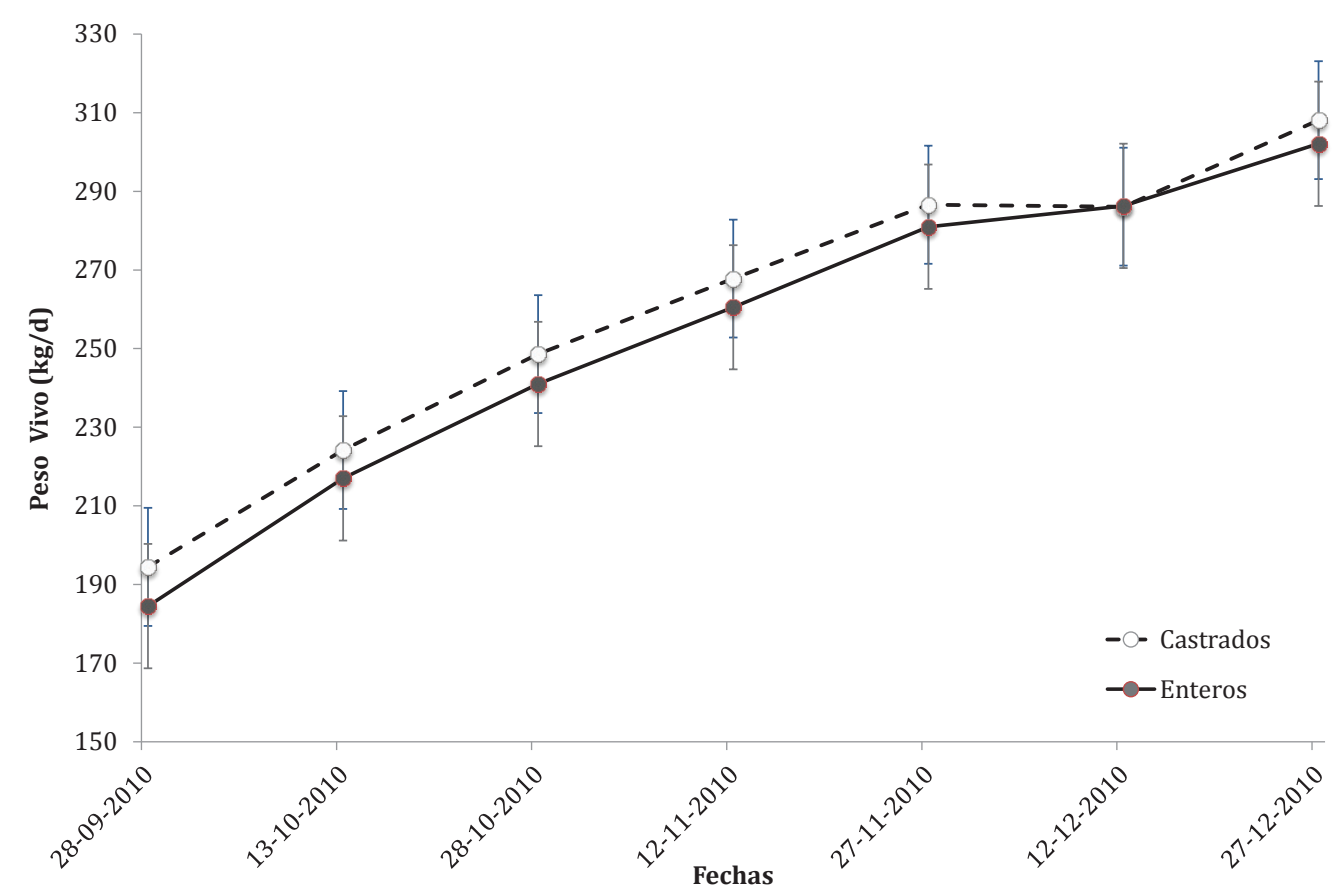

Figura 1.Evolución de la ganancia de peso vivo de los terneros durante el período de estudio (Las barras representan el error estándar de la media).

Figure 1.Evolution of body weight gain of calves during the study period. (The lines represent the standard error of the mean). 


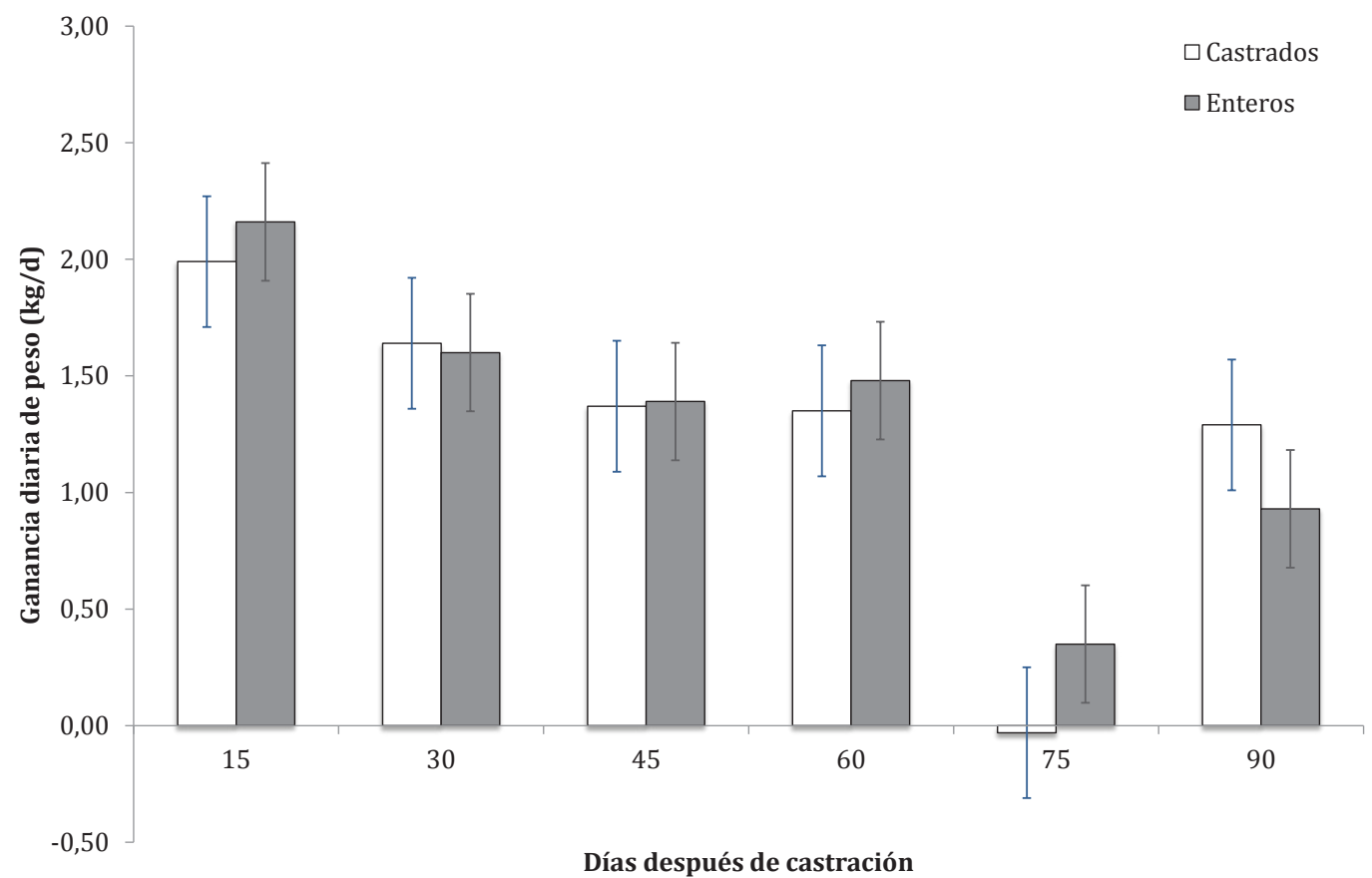

Figura 2.Ganancia de peso diaria promedio de los terneros en el estudio (Las barras representan el error estándar de la media. No se observaron diferencias estadísticas entre los tratamientos dentro delos periodos).

Figure 2. Average daily gain of calves under study (The lines represent the standard error of the mean. There was no statistical difference between treatments within periods).

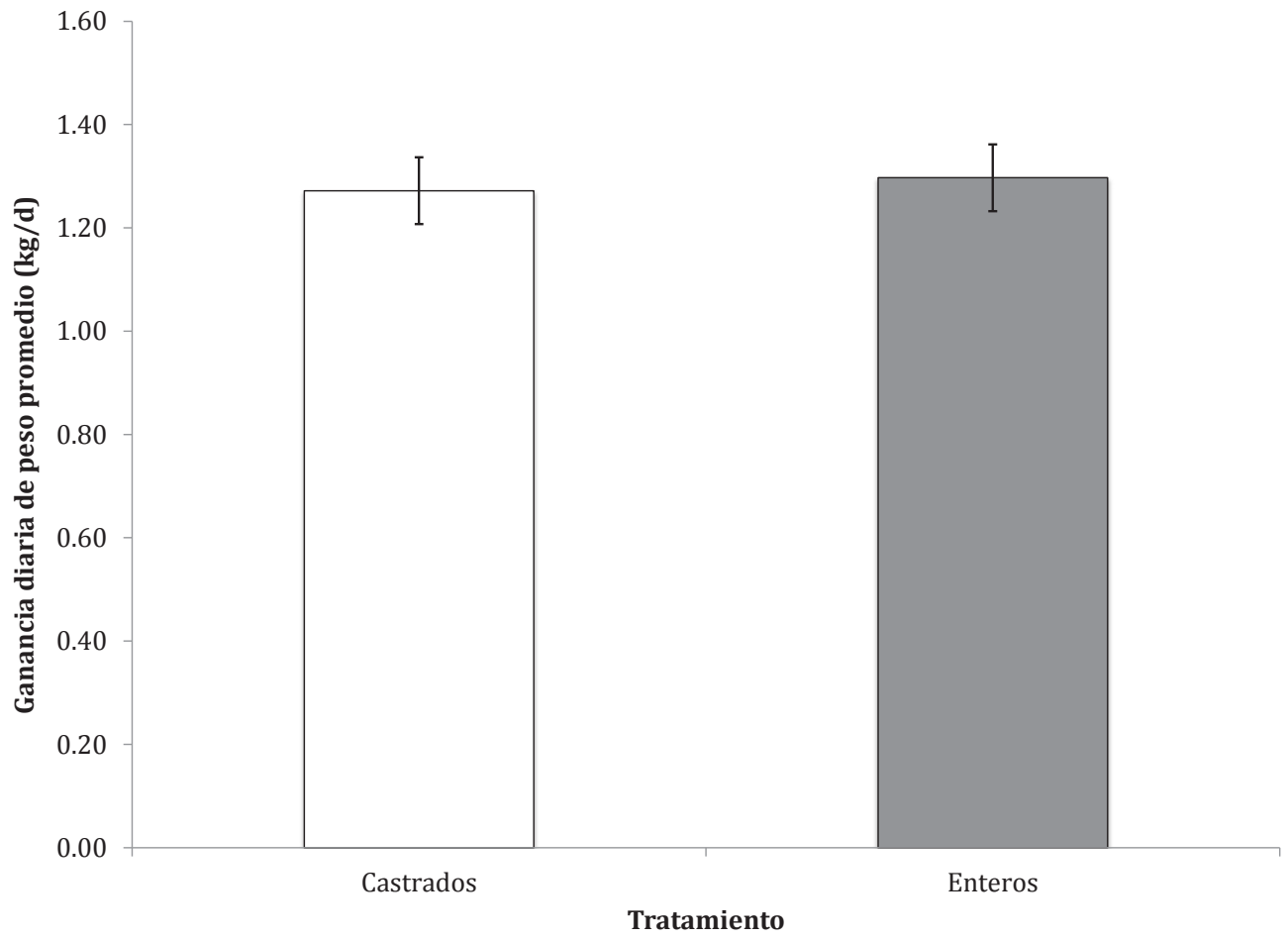

Figura 3.Ganancia de peso diaria promedio para el estudio (90 días) según tratamiento (Las barras representan el error estándar de la media).

Figure 3. Mean average daily gain for the whole study (90 days) by treatment. (The lines represent the standard error of the mean). 
FDN, lo que explicaría las ganancias de peso observadas, las que fueron superiores a las reportadas por Goic (2005b) de 0,6 kg/d en el área del llano central de la Región de Los Lagos bajo condiciones similares. Por otra parte, las GDP fueron levemente inferiores a los valores promedio de 8 años reportados por el mismo autor de $1,63 \mathrm{~kg} / \mathrm{d}$ para sistemas de recría-engorda en la misma región. En este sentido, Siebald (2005), señala que las praderas mixtas compuestas por varias especies presentan ventajas relacionadas con un buen balance de nutrientes, una menor incidencia de plagas y una mayor resistencia a condiciones adversas de climas. Sin embargo, uno de los problemas complejos es el manejo del pastoreo, debido a que en éste participan factores atribuibles a la pradera y al animal; $y$ a decisiones que toma el productor para llevarlo a cabo (Goic, 2005a). En tanto, la disminución de las ganancias de peso vivo en el tiempo en ambos grupos se explican por la curva natural de crecimiento de los animales, que presentan altas GDP durante el período postnatal, alcanzando su punto máximo al momento de la maduración, momento en el cual la GDP comienza a decrecer hasta que al animal alcanza su tamaño adulto (Hossner, 2005).

En lo referente a la menor GDP observada a los 75 días, esta podría haberse debido a una merma en la calidad y/o cantidad de forraje disponible, la cual no habría sido suficiente para satisfacer las demandas de los animales producto de las condiciones climáticas previas. Estas se caracterizaron por ser desfavorables para el desarrollo y crecimiento de la pradera, ya que durante el mes de noviembre hubo más frío de lo normal. Asimismo, las temperaturas ambientales y de suelo también fueron más bajas, lo que se tradujo en una menor acumulación de grados día respecto de un año normal. A lo anterior se suma un marcado déficit de las precipitaciones y una mayor radiación solar (Santibañez, 2010).

La práctica de castrar los machos bovinos destinados al sacrificio ha sido discutida en extenso desde hace varios años, particularmente en lo referente a la edad en la que es más conveniente realizar éste procedimiento así como también sus efectos sobre la calidad de la carne y el manejo de los animales. Actualmente existe consenso de que los animales enteros crecen más rápidamente (15 a 17\%) y utilizan el alimento más eficientemente (10 a 13\%) que los novillos a una edad o peso de faena que permita la expresión androgénica de la testosterona. También hay consenso de que los machos enteros producen canales de mayor rendimiento con menos grasa y más músculo (Field, 1971; Seideman et al., 1982). Por otra parte, la castración permite que los animales sean más dóciles o menos agresivos en su manejo, produzcan carne con un mayor grado de infiltración de grasa intramuscular, de mejor sabor, más jugosas y blandas que la de animales enteros (Rubio y Montiel, 1994; Knight et al., 1999a; Knight et al., 1999b; Bretschneider, 2009; Morón-Fuenmayor et al., 2010). Además, la carne de machos enteros se asocia a olores, sabores y un color de la carne indeseable (Seideman et al., 1982). Estos aspectos cobran gran relevancia en mercados en donde la calidad de la carne es premiada y los productores reciben el justo pago por la calidad producida. En el caso chileno, actualmente no existe un estímulo a producir carne de calidad o bien esto no es percibido a nivel de productores. De hecho, el ganado bovino es transado principalmente en ferias, incluidos los animales para engorda y animales gordos para faena, siendo las variables que determinan el precio fundamentalmente la edad, peso, conformación y fenotipo del animal. Asimismo, la normativa vigente de tipificación y calificación para el mercado interno considera la edad, cobertura grasa y contusiones como principales criterios de decisión.

Por otra parte, al igual que el destete, la castración es un estímulo altamente estresante para el animal, especialmente si no se efectúa a temprana edad. En efecto, la respuesta al estrés en el ganado castrado $\leq 6$ meses de edad tiende a ser menor que en el ganado de $>$ 6 meses (Bretschneider, 2005). Asimismo, Bretschneider (2009) plantea que durante el estrés se produce un incremento en los niveles sanguíneos de cortisol, una hormona que tiene propiedades inmunosupresivas y que predispone a enfermedades infecciosas, afectando con ello también la morbilidad del ganado. Los niveles de cortisol en sangre aumentan a medida que se incrementa la edad de castración. El mismo autor agrega que de los dos métodos de castración evaluados (cuchillo vs. banda de goma), la castración a cuchillo resultó ser más estresante para el animal (Bretschneider, 2009).

Según lo reportado por Bretschneider (2005), la pérdida de peso asociada a la castración se acentúa con la edad a la que ésta se efectúa, y se explicaría en parte por el menor consumo diario de alimento asociado a una menor actividad de desplazamiento y pastoreo. En este mismo sentido, Devant et al. (2012) llevaron a cabo un estudio en el cual compararon el comportamiento y respuesta productiva de animales enteros, castrados (a los 3 meses de edad) y toretes castrados a los 8 meses de edad. El peso inicial promedio de todos los animales fue de $220 \pm 22 \mathrm{~kg}$ y la edad promedio de 5,35 meses. Al comienzo del estudio los toretes pesaban más que novillos (226 vs. $208 \mathrm{~kg}$ ). Sin embargo, los toretes castrados a los 8 meses presentaron una menor ganancia de peso que los novillos y animales enteros durante los 15 días siguientes a la castración, en tanto, no hubo diferencias entre castrados y enteros en el mismo período. No obstante, estas diferencias desaparecen al considerar el período completo de estudio ( 5 a 10 meses de edad), lo que concuerda con lo observado en la presente investigación.

El momento en que se efectúa la castración depende de una serie de factores que incluyen aspectos tales 
como la filosofía del productor, condiciones climáticas, disponibilidad de la mano de obra, demandas del mercado e infraestructura adecuada, entre otros (Imler et al., 2013). En Chile, así como en muchas otras partes del mundo, una gran mayoría de los productores cree que el retraso de la castración mejora el crecimiento de los terneros en comparación con los terneros castrados poco después del nacimiento; y dado que a los productores se les paga en función del peso, el dejarlos enteros cobra mayor relevancia para ellos. Sin embargo, la mayor parte de la literatura existente indica que la castración de los terneros poco después del nacimiento tiene un efecto mínimo o bien ningún efecto sobre el peso al destete (Bailey et al., 1966; Glimp et al., 1971; Looper et al., 2005). Esto último aún cuando, la edad a la pubertad varía entre razas fluctuando esta entre las 33 y 53 semanas. En la literatura se ha reportado que los toros Hereford alcanzan la pubertad entre las 39 a 52 semanas en tanto los toros Charolais de 33 a 53 y los Holstein de 39 a 41 (Bagu et al., 2006).

Como se señaló anteriormente, la mayor ganancia de peso de los toros en comparación a los novillos se explica por la actividad androgénica de la testosterona. En este sentido, Rawlings et al. (1972) indican que los niveles de testosterona en el plasma en animales enteros es altamente variable, pero que generalmente estos aumentan desde valores prácticamente indetectables al momento del nacimiento a un promedio de $3,73 \mathrm{ng} / \mathrm{ml}$ a los 11 meses de edad, seguido por una fuerte caída a $0,50 \mathrm{ng} / \mathrm{ml}$ a los 12 meses. Otros estudios señalan la existencia de dos períodos de aumento en la secreción de gonadotrofinas, coincidiendo el segundo de ellos con la pubertad. Así entonces, los niveles de testosterona en toros aumentan de forma importante a partir del $5^{\circ}$ mes (Schams et al., 1981). Lo anterior coincide con lo reportado por Bagu et al. (2006) quienes además señalan una caída de los niveles de testosterona durante el $6^{\circ}$ y $7^{\circ}$ mes para nuevamente alcanzar valores similares a los observados en el $5^{\circ}$ mes en el $8^{\circ}$ mes. En tanto Rawlings et al. (1978) señalan que los niveles de testosterona son bajos durante el primer mes y aumentan gradualmente hasta el $7^{\circ}$ mes, alcanzando valores de un animal adulto a los 10 meses de edad, lo que coincide con lo señalado por Amann y Walker (1983). Basándose en lo antes expuesto es posible señalar que el deseado efecto androgénico no sería efectivo sino al menos hasta pasados los 6 meses de edad del animal. No obstante lo anterior, estos beneficios se diluirían al realizar la castración tardía (post-puberal), principalmente a causa del estrés y dolor que ésta provoca en el animal. En tanto, los datos del presente estudio no reflejan ventajas de los animales enteros por sobre los castrados, al menos para el período estudiado. En consecuencia, no se justificaría el postergar esta labor, ya que con ello se aumentan los riesgos para los operarios y los animales, reduciendo además su bienestar.

\section{CONCLUSIONES}

Bajo las condiciones en las que se desarrolló el estudio la castración no resultó en desventajas en la ganancia de peso diario cuando se le comparó con animales enteros. Por ello, no se justifica posponer la castración a una etapa post-pubertal y se recomienda hacerlo idealmente antes de los tres meses de edad.

\section{REFERENCIAS}

AMANN, R. P., WALKER, O. A. 1983. Changes in the PituitaryGonadal Axis Associated with Puberty in Holstein Bulls. Journal of Animal Science 57: 433-442.

ANRIQUE, R., MOLINA, X., ALFARO, M., SALDAÑA, R. 2014. Composición de alimentos para el ganado bovino. $4^{\text {ta }} \mathrm{ed}$. Consorcio Lechero - Universidad Austral de Chile.

BAGU, E. T., COOK, S., GRATTON, C. L., RAWLINGS, N. C. 2006. Postnatal changes in testicular gonadotropin receptors, serum gonadotropin, and testosterone concentrations and functional development of the testes in bulls. Reproduction 132: 403-411.

BAILEY, C. M., PROBERT, C. L., BOHMAN, V. R. 1966. Growth Rate, Feed Utilization and Body Composition of Young Bulls and Steers. Journal of Animal Science 25: 132-137.

BRETSCHNEIDER, G. 2005. Effects of age and method of castration on performance and stress response of beef male cattle: A review. Livestock Production Science 97: 89-100.

BRETSCHNEIDER, G. 2009. Castración de Terneros: Tradición versus Eficiencia. Revista electrónica de Veterinaria 10(7): 3 .

DEVANT, M., MARTI, S., BACH, A. 2012. Effects of castration on eating pattern and physical activity of Holstein bulls fed high-concentrate rations under commercial conditions. Journal of Animal Science 90: 4505-4513.

DUFF, G. C., GALYEAN, M. L. 2007. BOARD-INVITED REVIEW: Recent advances in management of highly stressed, newly received feedlot cattle. Journal of Animal Science 85: 823-840.

FIELD, R. A. 1971. Effect of Castration on Meat Quality and Quantity. Journal of Animal Science 32: 849-858.

FISHER, A. D., KNIGHT, T. W., COSGROVE, G. P., DEARTH, A. F., ANDERSON, C. B., DUGANZICH, D. M., MATTHEWS, L. R. 2001. Effects of surgical or banding castration on stress resonses and behaviour of bulls. Australian Veterinary Journal 79: 279-284.

GLIMP, H. A., DIKEMAN, M. E., TUMA, H. J., GREGORY, K. E., CUNDIFF, L. V. 1971. Effect of Sex Condition on Growth and Carcass Traits of Male Hereford and Angus Cattle. 
Journal of Animal Science 33: 1242-1247.

GOIC, L. 2005a. Manejo de pastoreo en praderas perennes. In: Catrileo A. S. (Ed.), Producción y Manejo de Carne Bovina en Chile No. Coleción Libros INIA $\mathrm{N}^{\circ} 16$. Instituto de Investigaciones Agropecuarias, Ministerio de Agricultura, Temuco. pp. 95-114.

GOIC, L. 2005b. Sistemas Productivos para la zona sur (X región). In: Catrileo A. S. (Ed.), Producción y Manejo de Carne Bovina en Chile No. Coleción Libros INIA N¹6. Instituto de Investigaciones Agropecuarias, Ministerio de Agricultura, Temuco, pp. 405-433.

HOCQUETTE, J. F., BOTREAU, R., LEGRAND, I., POLKINGHORNE, R., PETHICK, D. W., LHERM, M., PICARD, B., DOREAU, M., TERLOUW, E. M. C. 2014. Win-win strategies for high beef quality, consumer satisfaction, and farm efficiency, low environmental impacts and improved animal welfare. Animal Production Science 54: 1537-1548.

HOSSNER, K. L. 2005. Hormonal regulation of farm animal growth. CABI Publishing, Cambridge, MA. USA.

IMLER, A., THRIFT, T., HERSOM, M. J., YELICH, J. 2013. Effect of Age at Castration on Beef Calf Performance, University of Florida, Florida.

JAGO, J. G., MATTHEWS, L. R., TRIGG, T. E., DOBBIE, P., BASS, J. J. 1999. The effects of inmuno-castration seven weeks before slaughter on behaviour, growth and meat quality of post-pubertal bulls. Animal Science 68: 163 - 177.

KNIGHT, T. W., COSGROVE, G. P., DEATH, A. F., ANDERSON, C. B. 1999a. Effect of interval from castration of bulls to slaughter on carcass characteristics and meat quality. New Zealand Journal of Agricultural Research 42: 269277.

KNIGHT, T. W., COSGROVE, G. P., LAMBERT, M. G., DEATH, A. F. 1999b. Effects of method and age at castration on growth rate and meat quality of bulls. New Zealand Journal of Agricultural Research 42: 255-268.

LOOPER, M. L., BURKE, J. M., MCBETH, L. J., KREHBIEL, C. R., FLORES, R., ROSENKRANS, C. F., AIKEN, G. E. 2005. Growth, Feedlot Performance, and Carcass Characteristics of Angus and Charolais-Sired Calves Castrated at Birth or Weaning, University of Arkansas, Arkansas.

MORÓN-FUENMAYOR, O., ARAUJO-FEBRES, O., PIETROSEMOLI, S., GALLARDO, N., SULBARÁN, B., PEÑA, J. 2010.
Efecto de la castración sobre la composición físicoquímica y características sensoriales en carne de bovinos mestizos comerciales. Revista Facultad Agronomía. 2010: 594-606.

MOSHER, R. A., WANG, C., ALLEN, P. S., COETZEE, J. F. 2013. Comparative effects of castration and dehorning in series or concurrent castration and dehorning procedures on stress responses and production in Holstein calves. Journal of Animal Science 91: 4133-4145.

MÜLLER-LINDENLAUF, M., DEITTERT, C., KÖPKE, U. 2010. Assessment of environmental effects, animal welfare and milk quality among organic dairy farms. Livestock Science 128: 140-148.

NRC. 2000. Nutrient Requirement of Beef Cattle, update 2000. National Academy Press, Washington DC.

RAWLINGS , N. C., FLETCHER , P. W., HENRICKS, D. M., HILL, J. R. 1978. Plasma Luteinizing Hormone (LH) and Testosterone Levels during Sexual Maturation in Beef Bull Calves. Biology of Reproduction 19: 1108-1112.

RAWLINGS, N. C., HAFS, H. D., SWANSON, L. V. 1972. Testicular and Blood Plasma Androgens in Holstein Bulls from Birth through Puberty. Journal of Animal Science 34: 435-440.

RUBIO, N., MONTIEL, N. 1994. Efecto comparativo sobre la ganancia de peso de dos agentes anabólicos en mestizos Bos indicus enteros y castrados a pastoreo, Universidad Rafael Urdeneta, Facultad de Ciencias Veterinarias, Universidad del Zulia. Maracaibo.

SANTIBAÑEZ, P. 2010. Boletín Agroclimático Regional. Región de Los Lagos. Período Diciembre, www.agroclima.cl.

SCHAMS, D., SCHALLENBERGER, E., GOMBE, S., KARG, H. 1981. Endocrine patterns associated with puberty in male and female cattle. Journal of Reproduction and Fertility. Supplement 30: 103-110.

SEIDEMAN, S. C., CROSS, H. R., OLTJEN, R. R., SCHANBACHER, B. D. 1982. Utilization of the Intact Male for Red Meat Production: A Review. Journal of Animal Science 55: 826-840.

SIEBALD, E. 2005. Praderas naturalizadas para la producción de carne bovina. In: Catrileo A. S. (Ed.), Producción y Manejo de Carne Bovina en Chile No. Coleción Libros INIA $\mathrm{N}^{\circ} 16$. Instituto de Investigaciones Agropecuarias. Ministerio de Agricultura, Temuco, pp. 61-74. 
